\title{
Ambiente obesogênico universitário: achados de uma cidade brasileira de grande porte
}

\author{
University obesogenic environment: findings from a \\ brazilian large city
}

\author{
Bianca Elidia Sodré', Maria Alvim Leite ${ }^{2}$, Mirella Lima Binoti ${ }^{3}$ \\ 'Nutricionista, formada pela universidade federal de Juiz de Fora \\ ${ }^{2}$ Doutoranda do Programa de Pós-Graduação em Saúde Coletiva da Universidade de São \\ Paulo (USP) \\ ${ }^{3}$ Universidade Federal de Viçosa
}

Email para contato: Mirella Lima Binoti - mirella.binoti@ufv.br

\begin{abstract}
Resumo
Introdução: O contexto em que as populações vivem pode influenciar negativamente a qualidade da sua alimentação, constituindo os chamados ambientes obesogênicos. O objetivo desse estudo foi avaliar os tipos de comércios existentes e a disponibilidade de alimentos comercializados em relação ao seu grau de processamento industrial, dentro e ao redor da Universidade Federal de Juiz de Fora. Métodos: Trata-se de estudo exploratório, no qual foram avaliados todos os comércios de alimentos localizados dentro de um buffer de raio de 500 metros. Foram coletados dados de latitude e longitude dos estabelecimentos e informações dos alimentos comercializados. Os estabelecimentos foram agrupados de acordo com suas características e de acordo com grau de processamento da maioria dos alimentos vendidos. Utilizou-se o Teste Exato de Fisher para testar a associação entre tipo de estabelecimento e grau de processamento da predominância dos alimentos comercializados. Para avaliar possíveis aglomerações, foram feitos mapas de densidade de Kernel. Resultados: Foram avaliados 39 comércios que ofereciam, predominantemente, alimentos ultraprocessados e aglomeravam-se no centro da área estudada. Conclusão: Observa-se que o ambiente alimentar no qual os frequentadores da instituição estão expostos pode ser classificado como ambiente não promotor de uma alimentação saudável.
\end{abstract}

Palavras-chave: Alimentos Industrializados. Universidades. Saúde Pública.

\begin{abstract}
Introduction: The environment may influence negatively the quality of individuals' food consumption, constituting the so-called obesogenic environments. The aim of the study was to evaluate the types of food retailers and the availability of foods according to the degree of industrial processing, inside and around the Federal University of Juiz de Fora, at Juiz de Fora, Brazil. Methods: This exploratory study evaluated all food retailers located within a buffer with a 500 meters radius. Data of latitude and longitude of the establishments and information about the sold foods were collected. The establishments were grouped according to their characteristics and the processing level of the most part of the marketed foods. Fisher's exact test were used to test the association between the type of
\end{abstract}


establishment and the degree of processing of the most part of the sold food. To evaluate agglomerations, Kernel density maps were used. Results: The researchers evaluated 39 food retailers, offering predominantly ultra-processed food and concentrated in the center of the studied area. Conclusion: It is observed that the food environment in which students and university employees are exposed can be characterized with a low nutritional quality.

Keywords: Industrialized Foods. Environment. Public Health.

\section{INTRODUÇÃO}

O ambiente pode exercer uma influência negativa para a qualidade da alimentação e para a prática de atividade física, constituindo os chamados ambientes obesogênicos ${ }^{1}$. Esses ambientes são aqueles que expõem os indivíduos a locais que fornecem alimentos densamente energéticos e de baixo valor nutricional e, ao mesmo, tempo induzem ao sedentarismo, por não oferecerem espaços apropriados à prática de exercícios e à mobilidade ativa² .

Medir e estudar o ambiente alimentar são medidas fundamentais para a compreensão da influência do mesmo no aumento da prevalência da obesidade, e assim, auxiliar na elaboração de políticas públicas eficientes na promoção da saúde3. Além disso, por ser uma temática relativamente recente para a ciência da nutrição, novos estudos podem de contribuir com a consolidação de medidas eficientes para mensuração desses ambientes ${ }^{4}$.

De maneira complementar à influência do ambiente, as modificações nos hábitos alimentares e no estilo de vida da população brasileira vêm sendo percebidas cada vez com maior intensidade. Na última década, foram notadas mudanças significativas na constituição da base alimentar da população ${ }^{5}$, como um aumento do número de refeições realizadas fora de domicílio ${ }^{6}$. Os gastos mensais com essas refeições comprometeram aproximadamente $32,8 \%$ da renda das famílias de acordo com a última Pesquisa de Orçamentos Familiares (POF), realizada entre 2018 e $2019^{6}$.

Essa transformação ocorreu devido à necessidade de se adequar aos novos padrões de comportamento, como as jornadas de trabalho cada vez mais intensas, lazer, estudos, entre outros ${ }^{7,8}$. Com o aumento do consumo de alimentos fora de domicílio e de estabelecimentos que prestam esse serviço ${ }^{9}$, aumenta também a chance das pessoas consumirem, em maiores quantidades, produtos com baixos valores nutricionais e elevado teor calórico, em comparação às refeições realizadas em ambiente domiciliar ${ }^{10,11}$. Em discordâncias com tais práticas e a partir de evidências 
científicas, o Guia Alimentar para a População Brasileira propõe que alimentos in natura ou minimamente processados sejam a base da alimentação e, além disso, incentiva o preparo de alimentos em domicílio e o desenvolvimento de habilidades culinárias ${ }^{8}$.

Instituições de ensino ou locais de trabalho, como as universidades, geralmente têm como característica um ambiente alimentar pouco diversificado, e, devido à densa carga horária de trabalho ou estudo, as pessoas que ali frequentam tendem a optar por consumirem produtos industrializados e refeições rápidas encontradas nesses ambientes e em suas proximidades ${ }^{12}$. Por essa razão, alguns estudantes e funcionários universitários não apresentam uma rotina alimentar saudável, demonstrando um consumo de alimentos industrializados cada vez maior, além de baixa ingestão de frutas e hortaliças, o que significa, muitas vezes, baixo aporte de fibras, vitaminas e minerais ${ }^{13}$.

No entanto, há uma escassez de estudos que investiguem a qualidade dos alimentos ofertados dentro e nas proximidades dos centros acadêmicos, principalmente os brasileiros. Diante do exposto, o objetivo deste trabalho foi avaliar a disponibilidade de alimentos comercializados em relação ao seu grau de processamento industrial, e os tipos de comércio existente no entorno de uma instituição federal de ensino superior de uma cidade brasileira de grande porte.

\section{MÉTODO}

Trata-se de estudo exploratório realizado dentro e no entorno da Universidade Federal de Juiz de Fora (UFJF), em Juiz de Fora, Minas Gerais, Brasil. A cidade é classificada como porte grande devido às suas funções econômica e administrativa e seu volume populacional. A área urbana do município é composta por $317,740 \mathrm{~km}^{2}$. Em 2017, o Produto Interno Bruto (PIB) per capita era de R\$ 28.355,07; o salário médio mensal dos trabalhadores formais de 2,5 salários-mínimos; e o Índice de Desenvolvimento Humano Municipal (IDHM) de 0,778. A população estimada em 2019 era de 568.873 habitantes $^{14,15}$.

Foram avaliados todos os comércios localizados dentro de uma área circular de raio de 500 metros, traçado com auxílio do Software Autocad ${ }^{\circledR}$ (Computer Aided Design), a partir do centroide, localizado na Reitoria da UFJF. Essa medida foi definida considerando o usual percurso realizado a pé pelos frequentadores da instituição, localizada na região oeste do município, na qual circulam cerca de 20.000 pessoas 
diariamente, levando em conta todos os alunos matriculados e os servidores ${ }^{16}$ da Universidade.

A coleta dos dados geográficos de latitude e longitude dos estabelecimentos foi realizada entre fevereiro e março de 2016, quando foram percorridas todas as ruas e analisados todos os estabelecimentos comerciais dentro do referido raio. As coordenadas foram coletadas com auxílio do aplicativo para aparelhos androides Coletap ${ }^{\circledR}$, posicionando-se em frente aos estabelecimentos pesquisados.

A avaliação do tipo de estabelecimento e dos alimentos comercializados foi realizada por meio de observação e registro dos dados em uma lista de verificação extraída de um instrumento validado, desenvolvido por Leite et al. ${ }^{17}$. Esse inventário de alimentos comercializados, composto por 33 itens, baseou-se nos grupos descritos no Guia de Harvard ${ }^{16}$ e nos diferentes graus de processamento industrial dos alimentos $^{8}$. Os estabelecimentos comerciais foram classificados em três categorias, de acordo com seu propósito de comercialização de alimentos: 1) açougues e hortifrútis; 2) bares, lanchonetes, restaurantes, ambulantes, cantinas e bomboniéres; 3) mercearias, padarias e supermercados. Os alimentos, considerados marcadores, foram registrados como "comercializados" ou "não comercializados".

Posteriormente, a partir dos dados obtidos, os alimentos foram classificados e divididos em três classes de acordo com o seu grau de processamento, segundo a Nova Classificação dos Alimentos (NOVA), proposta por Monteiro e colaboradores ${ }^{19}$. Não foi considerada a categoria dos ingredientes culinários. Considerando a predominância dos alimentos ofertados em cada estabelecimento, os comércios foram agrupados como: a) maior disponibilidade de alimentos in natura ou minimamente processados (AMP); b) maior disponibilidade de alimentos processados (AP); c) maior disponibilidade de alimentos ultraprocessados (AUP).

No primeiro grupo (AMP), se enquadraram os estabelecimentos que comercializavam predominantemente alimentos que sofreram mínimas ou nenhuma alteração antes de serem adquiridos, como folhas, frutas, carnes, ovos e leites, classificados como in natura ou minimamente processados ${ }^{19}$.

No segundo grupo (AP) estão aqueles que vendem principalmente alimentos processados, que são produtos feitos a partir de alimentos in natura com adição de óleos, gordura, açúcares e sal, utilizados como ingredientes culinários. São exemplos os pães feitos à base de farinha de trigo sem gordura hidrogenada ou aditivos, conservas de frutas e legumes, queijos entre outros ${ }^{19}$. 
No terceiro grupo (AUP), estão os estabelecimentos que disponibilizam maiores quantidades de alimentos ultraprocessados, que são aqueles prontos para o consumo, cuja maior parte da composição representa substâncias processadas extraídas de alimentos in natura ou sintetizadas em laboratórios. São representantes desse grupo: biscoitos doces e salgados industrializados, chips, doces como balas, pirulitos e chicletes, bebidas como refrescos em pó, refrigerantes e bebidas lácteas $\operatorname{adoçadas~}^{19}$.

Quando um estabelecimento apresentava disponíveis as mesmas quantidades de alimentos de diferentes graus de processamentos, optou-se por classificá-lo de acordo com o maior grau. Tal procedimento foi considerado no tratamento dos dados prévio às análises estatísticas.

As análises estatísticas numéricas foram feitas no Statistical Package for the Social Science (SPSS), versão 20.0 para Windows, e as operações espaciais no Sistema de Informações Geográficas (SIG) QGIS 2.8.6.

As variáveis foram descritas por meio de frequência absoluta (n) e frequência relativa (\%). Utilizou-se o Teste Exato de Fisher para testar a associação entre o tipo de estabelecimento e o grau de processamento da predominância dos alimentos comercializados. $\mathrm{O}$ tamanho do efeito foi avaliado pelo $\mathrm{V}$ de Cramer, utilizando as classificações pequeno $(0,10$ a 0,30$)$, moderado $(0,30$ a 0,50$)$ ou elevado (acima de 0,50)20. O nível de significância de p<0,05 (5\%) foi adotado nos procedimentos analíticos para a interpretação dos resultados obtidos. Para representação gráfica dos resultados, foram confeccionados mapas de densidade Kernel (mapas de calor).

\section{RESULTADOS}

O estudo contou com um total de 39 pontos de venda de alimentos, situados dentro da região estudada (Figura 1).

Com relação às categorias de comércios investigadas, a maioria, 79,49\% (n= 31), foi constituída por bares, lanchonetes, restaurantes, ambulantes, cantinas e bomboniéres (categoria 2$)$. Outros $12,82 \%(n=5)$ eram compostos por mercearias, padarias e supermercados (categoria 3) e apenas $7,69 \%(n=3)$ eram formados por açougues e hortifrútis (categoria 1 ).

Quanto à distribuição dos grupos de alimentos que compuseram a lista de verificação em relação às diferentes categorias de estabelecimentos (Tabela 1), pode-se observar que na categoria 1 (açougues e hortifrútis), frequentemente foram 
encontrados alimentos como: ovos, bebidas açucaradas, enlatados, hortaliças, frutas, leguminosas e doces como balas, chicletes e pirulitos.

Figura 1 - Representação do local do estudo e arredores da Universidade Federal de Juiz de Fora (UFJF). Juiz de Fora (MG), 2016.

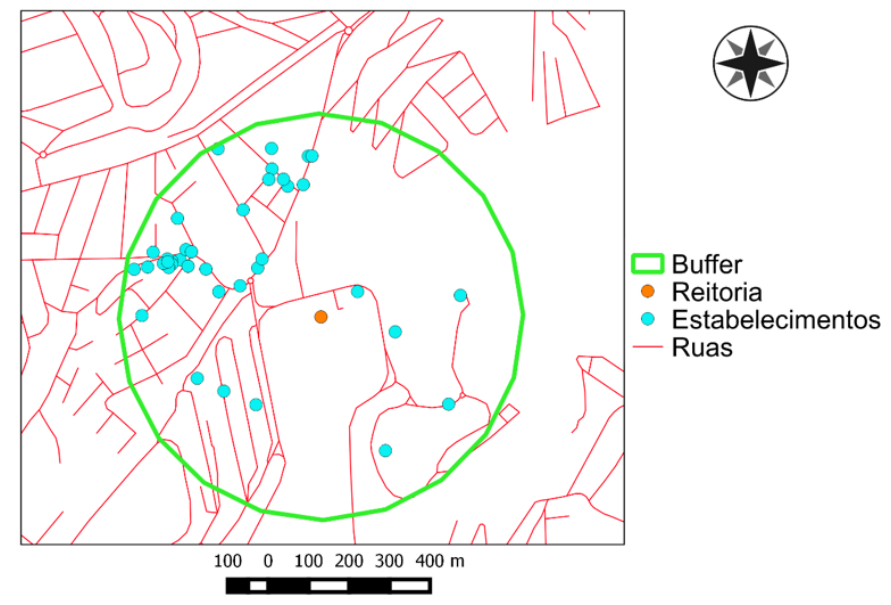

Tabela 1 - Disponibilidade dos grupos de alimentos nas diferentes categorias de comércios dentro e ao redor de uma universidade pública. Juiz de Fora (MG), 2016.

\begin{tabular}{|c|c|c|c|c|}
\hline \multirow[b]{2}{*}{ Grupos de alimentos } & \multicolumn{4}{|c|}{ Categorias de Comércios* } \\
\hline & $\begin{array}{c}1 \\
\text { n (\%) }\end{array}$ & $\begin{array}{c}2 \\
\text { n (\%) }\end{array}$ & $\begin{array}{c}3 \\
\text { n (\%) }\end{array}$ & $\begin{array}{l}\text { Total } \\
\text { n (\%) }\end{array}$ \\
\hline Bebidas açucaradas & $2(66,7)$ & $31(100)$ & $4(80,0)$ & $37(94,9)$ \\
\hline Biscoito sem Recheio & $1(33,3)$ & $12(38,7)$ & $4(80,0)$ & $17(43,6)$ \\
\hline Biscoito com Recheio & $0(0,0)$ & $11(35,5)$ & $4(80,0)$ & $15(38,5)$ \\
\hline Bolos & $0(0,0)$ & $5(16,1)$ & $3(60,0)$ & $8(20,5)$ \\
\hline Cereais Prontos & $0(0,0)$ & $0(0,0)$ & $2(40,0)$ & $2(5,1)$ \\
\hline Cereais Farináceos & $2(66,7)$ & $0(0,0)$ & $3(60,0)$ & $5(12,8)$ \\
\hline Massas Prontas & $1(33,3)$ & $1(3,2)$ & $3(60,0)$ & $5(12,8)$ \\
\hline Óleo e Gorduras & $1(33,3)$ & $1(3,2)$ & $4(80,0)$ & $6(15,4)$ \\
\hline Oleaginosas e sementes & $0(0,0)$ & $0(0,0)$ & $1(20,0)$ & $1(2,6)$ \\
\hline Ovos & $3(100,0)$ & $1(3,2)$ & $5(100,0)$ & $9(23,1)$ \\
\hline Pães & $0(0,0)$ & $2(6,5)$ & $3(60,0)$ & $5(12,8)$ \\
\hline Salgados assados & $0(0,0)$ & $17(54,8)$ & $2(40,0)$ & $19(48,7)$ \\
\hline Cereais Integrais & $0(0,0)$ & $0(0,0)$ & $1(20,0)$ & $1(2,6)$ \\
\hline Chips & $0(0,0)$ & $13(41,9)$ & $4(80,0)$ & $17(43,6)$ \\
\hline Doces & $2(66,7)$ & $27(87,1)$ & $4(80,0)$ & $33(84,6)$ \\
\hline Sobremesas prontas & $0(0,0)$ & $18(58,1)$ & $3(60,0)$ & $21(53,9)$ \\
\hline Enlatados & $2(66,7)$ & $1(3,2)$ & $5(100,0)$ & $8(20,5)$ \\
\hline Frutas & $2(66,7)$ & $3(9,7)$ & $3(60,0)$ & $8(20,5)$ \\
\hline Hortaliças & $2(66,7)$ & $0(0,0)$ & $3(60,0)$ & $5(12,8)$ \\
\hline Leguminosas & $2(66,7)$ & $0(0,0)$ & $3(60,0)$ & $5(12,8)$ \\
\hline Leite e derivados & $0(0,0)$ & $1(3,2)$ & $4(80,0)$ & $5(12,8)$ \\
\hline Salgados Fritos & $0(0,0)$ & $17(54,8)$ & $2(40,0)$ & $19(48,7)$ \\
\hline Sanduíches & $0(0,0)$ & $12(38,7)$ & $3(60,0)$ & $15(38,5)$ \\
\hline Picolé & $0(0,0)$ & $10(32,3)$ & $3(60,0)$ & $13(33,3)$ \\
\hline Carnes não processadas & $1(33,3)$ & $5(16,1)$ & $1(20,0)$ & $7(18,0)$ \\
\hline Carnes Processadas & $1(33,3)$ & $4(12,9)$ & $1(20,0)$ & $6(15,4)$ \\
\hline Pescado fresco & $0(0,0)$ & $0(0,0)$ & $7(20,0)$ & $7(2,6)$ \\
\hline Queijos não processados & $0(0,0)$ & $6(19,4)$ & $4(80,0)$ & $10(25,6)$ \\
\hline Queijos Processados & $0(0,0)$ & $1(3,2)$ & $3(60,0)$ & $4(10,3)$ \\
\hline Refeições prontas & $0(0,0)$ & $9(29,0)$ & $1(20,0)$ & $10(25,6)$ \\
\hline
\end{tabular}

* Categoria 1: açougues e hortifrútis; Categoria 2: bares, lanchonetes, restaurantes, ambulantes, cantinas e bomboniéres; Categoria 3: mercearias, padarias e supermercados. 
Relativo à categoria 2 (bares, lanchonetes, restaurantes, ambulantes, cantinas e bomboniéres), em mais da metade dos estabelecimentos foram encontrados alimentos como bebidas artificiais açucaradas, salgados assados e fritos, sobremesas prontas e doces. Nessa categoria, alguns restaurantes e cantinas, além de oferecerem a opção de lanches, forneciam também refeições prontas, compostas por saladas, prato principal, guarnições e acompanhamentos.

Já a categoria 3 (mercearias, padarias e supermercados), apresentou em mais da metade dos estabelecimentos alimentos como bebidas artificiais açucaradas, biscoitos com e sem recheios, chips, bolos processados, pães processados, doces, sobremesas prontas, picolé, massas prontas, sanduíches, queijos, enlatados, leite e derivados, leguminosas, hortaliças, frutas, ovos, cereais farináceos, óleos e gorduras.

Já em relação ao agrupamento dos estabelecimentos de acordo com o grau de processamento da maioria dos alimentos comercializados, $28,21 \%(n=11)$ se inseriram no grupo AMP, 25,64\% $(n=10)$ no grupo AP e 46,15\% $(n=18)$ no grupo AUP.

Foi observada associação estatisticamente significante entre o tipo de comércio e o grau de processamento dos alimentos comercializados (Tabela 2).

Tabela 2 - Oferta de alimentos minimamente processados, processados e ultraprocessados nas diferentes categorias de comércios dentro e ao redor de uma universidade pública. Juiz de Fora (MG), 2016.

\begin{tabular}{|c|c|c|c|c|c|c|}
\hline & \multicolumn{4}{|c|}{$\begin{array}{l}\text { Grupos de acordo com o grau de processamentos } \\
\text { dos alimentos comercializados* }\end{array}$} & \multirow{2}{*}{ p-valor } & \multirow{2}{*}{ V-Cramer } \\
\hline & AMP & AP & AUP & Total & & \\
\hline \multicolumn{7}{|l|}{$\begin{array}{l}\text { Categorias de } \\
\text { comércios** }\end{array}$} \\
\hline 1 & 3 (100\%) & $\mathrm{O}(0,0 \%)$ & $\mathrm{O}(0,0 \%)$ & $3(100,0 \%)$ & & \\
\hline \multirow[t]{2}{*}{2} & $5(16,1 \%)$ & $8(25,8 \%)$ & $18(58,1 \%)$ & $31(100,0 \%)$ & & \\
\hline & & & & & $0,001^{a}$ & 0,43 \\
\hline 3 & $3(60,0 \%)$ & $2(40,0 \%)$ & $\mathrm{O}(0,0 \%)$ & $5(100,0 \%)$ & & \\
\hline Total & $11(28,2 \%)$ & $10(25,6 \%)$ & $18(46,2 \%)$ & 39 (100,0\%) & & \\
\hline
\end{tabular}

*AMP: locais que vendem predominantemente alimentos minimamente processados; AP: locais que vendem predominantemente alimentos processados; AUP: locais que vendem predominantemente alimentos ultra processados; ${ }^{* *}$ 1: açougues e hortifrútis; 2 : bares, lanchonetes, restaurantes, ambulantes, cantinas e bomboniéres; 3: mercearias, padarias e supermercados; a Associação estatisticamente significante, $\mathrm{p}<0,05$, através do Teste Exato de Fisher. 
O valor da estatística do Teste Exato de Fisher foi de 12,536, com valor de probabilidade associado de 0,001. Sob o ponto de vista prático, o V de Cramer obtido de 0,43 sugere que esta associação é de moderada magnitude. Pode-se dizer que $18,5 \%$ da predominância da comercialização de alimentos ultraprocessados pode ser explicada pelo tipo de comércio. Verificou-se que 100\% dos açougues e hortifrútis comercializavam predominantemente alimentos minimamente processados. Os locais que comercializavam por predominância alimentos ultraprocessaodos eram os bares, lanchonetes, restaurantes, ambulantes, cantinas e bomboniéres. Já as mercearias, padarias e supermercados comercializam, majoritariamente, alimentos minimamente processados e processados.

A análise das aglomerações dos comércios na região estudada permitiu visualizar a maior presença de estabelecimentos com venda predominante de alimentos ultraprocessados, representando quase a metade do total de estabelecimentos, principalmente dentro da universidade, onde se encontram as cantinas. Nessa análise, as cores mais fortes (escuras) representam maior concentração de pontos (Figura 2).

Figura 2 - Avaliação das aglomerações dos comércios com predominância de alimentos in natura ou minimamente processados, processados e ultraprocessados em um raio de $500 \mathrm{~m}$ em torno da Reitoria da Universidade Federal de Juiz de Fora (UFJF). Juiz de Fora (MG), 2016.

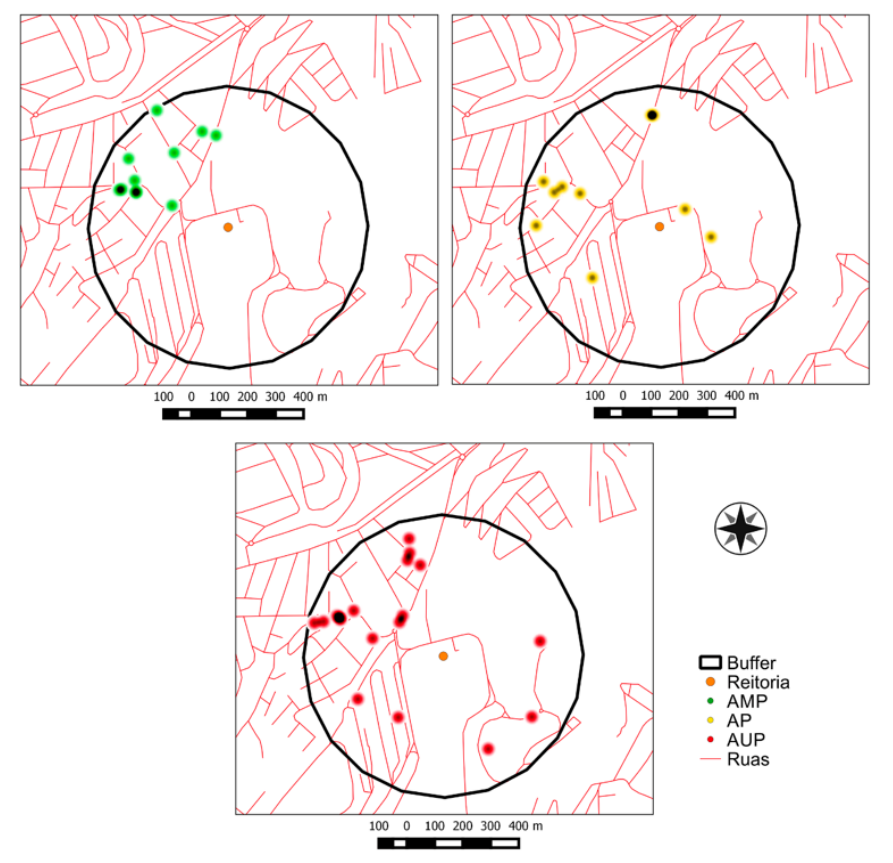




\section{DISCUSSÃO}

Nota-se que na terceira categoria de estabelecimentos (mercearias, padarias e supermercados) apesar de haver uma ampla oferta de alimentos ultraprocessados (Tabela 1), de acordo com o instrumento utilizado na pesquisa, os marcadores dos outros grupos de alimentos eram ainda mais presentes (Tabela 2).

Estudos ${ }^{21,22}$ mostram que em vizinhanças com baixa densidade e/ou variedade de estabelecimentos, os supermercados atuam como "oásis" para a compra de alimentos frescos e saudáveis como frutas, legumes e hortaliças. Por outro lado, devido à conveniência e ao baixo preço, são espaços atrativos para a compra de alimentos ultraprocessados ${ }^{21}$.

Os resultados encontrados no estudo confirmaram uma crescente oferta, em múltiplos espaços, de alimentos prontos para consumo10, notadamente mais caros e menos saudáveis em relação aos alimentos preparados em domicílio ${ }^{24,25}$. O público que a universidade recebe é formado, principalmente, por jovens e adultos. Nessas faixas etárias é possível constatar o hábito de consumir alimentos fora de domicílio como pizzas, refrigerantes, salgados fritos e assados ${ }^{9}$.

$\mathrm{Na}$ área estudada, os estabelecimentos comerciais em maior quantidade pertenciam à categoria 2 (bares, lanchonetes, restaurantes, ambulantes, cantinas e bomboniéres). Nesses locais, é comum a aquisição de alimentos ou preparações culinárias para consumo imediato, como salgados fritos e assados, bebidas açucaradas, doces e sobremesas prontas.

Berti et al., em seu estudo, verificaram que adultos mais jovens apresentaram maior consumo de ultraprocessados, hábitos alimentares não saudáveis, que na sua maioria são altamente calóricos e pobres em fibras, vitaminas e minerais, ressaltando a necessidade de intervenções principalmente nessa faixa etária, e indicando tendências futuras com implicações negativas na saúde dessa população ${ }^{26}$.

O ambiente alimentar no qual o indivíduo está inserido tem se mostrado importante ao influenciar em suas escolhas alimentares ${ }^{12,27}$. Sendo formado como uma teia, envolve características como quantidade de comércios presentes na área, tipos de estabelecimentos, produtos ofertados, preços, disponibilidade de gêneros alimentícios e de bebidas ${ }^{28}$. A partir das análises realizadas, constatou-se que quase metade $(46,2 \%, \mathrm{n}=18)$ dos estabelecimentos na área estuda comercializam 
predominantemente alimentos ultraprocessados. Outros estudos ${ }^{29,30}$, que tinham como objetivo avaliar alimentos comercializados e consumidos em ambientes universitários, também constataram que esses locais apresentavam limitações de oferta de alimentos saudáveis e grandes ofertas de alimentos ricos em ingredientes refinados, calorias, açúcares, sal e gorduras.

A indústria está cada vez mais interessada em produzir alimentos com alto teor de sal, gordura e açúcar com o objetivo de aumentar o tempo de prateleira e de tornar os produtos mais palatáveis, consequentemente tornando-os cada vez menos saudáveis ${ }^{8,29,31}$. Neste trabalho, foi verificado que os frequentadores da instituição estudada estão expostos a alimentos ricamente constituídos por esses três ingredientes, ao mesmo tempo em que existe uma baixa disponibilidade de alimentos saudáveis, promotores de uma alimentação saudável. A grande concentração e aglomeração de produtos processados e ultraprocessados no entorno da universidade podem contribuir para o prejuízo do estado nutricional dos seus frequentadores devido sua composição e seu modo de produção ${ }^{32}$, portanto, alimentos processados devem ser consumidos com moderação e os ultraprocessados devem ser evitados ${ }^{8}$. As atuais diretrizes do Ministério da Saúde ${ }^{8}$ reforçam que a base da alimentação deve ser composta por alimentos in natura ou minimamente processados.

Esse estudo utiliza uma metodologia previamente descrita ${ }^{17}$ e é um dos poucos estudos que se tem conhecimento que prioriza o grau de processamento industrial dos alimentos para investigação do ambiente alimentar. Por avaliar in loco todos os estabelecimentos presentes em uma área pré-estabelecida, o diagnóstico do ambiente alimentar construído tende a ser altamente preciso. Como limitação, temse que a investigação foi feita em uma região restrita, que não abarca todas as possibilidades de consumo dos indivíduos que convivem na região universitária. Buscou-se, porém, investigar áreas de maior circulação dos estudantes, professores e funcionários da instituição. A lista dos alimentos utilizada pode não conter todos os produtos presentes em cada um dos estabelecimentos, no entanto, por se tratar de um instrumento de pesquisa validado e já utilizado ${ }^{17}$, acredita-se que a ferramenta cumpre seu papel na avaliação ambiente alimentar.

\section{CONCLUSÃO}

Este estudo é um ponto de partida para que novas pesquisas sejam realizadas a fim de se investigar a qualidade dos alimentos ofertados dentro e próximos aos centros 
universitários. De acordo com os dados que foram encontrados, observa-se que o ambiente alimentar no qual os estudantes, professores e funcionários estão inseridos pode ser classificado ambiente não promotor de uma alimentação saudável, por incentivar e expor seus frequentadores ao consumo de alimentos ultraprocessados. Medidas de intervenção devem ser tomadas com intuito de melhorar a qualidade dos alimentos oferecidos e dos estabelecimentos alimentares situados dentro do campus e em seus arredores. Complementarmente, intervenções nutricionais de cunho educativo devem ser realizadas, de forma a esclarecer e informar os consumidores sobre a importância de inserir alimentos frescos e minimamente processados em suas refeições, visando à promoção da saúde por meio da alimentação saudável, diminuindo o risco de desenvolvimento de obesidade e outras doenças associadas.

\section{REFERÊNCIAS}

1. Swinburn B, Egger G, Razza F. Dissecting Obesogenic Environments: The Development and Application of a Framework for Identifying and Prioritizing Environmental Interventions for Obesity. Prev Med 1999; 29:563-570.

2. Matozinhos FP. Fatores ambientais e individuais associados à obesidade [tese]. Belo Horizonte: Escola de Enfermagem da Universidade Federal de Minas Gerais; 2015.

3. Glanz K. Measuring food environments: a historical perspective. American Journal of Preventive Medicine 2009; 36(4): S93-S98.

4. Glanz K, Sallis JF, Saelens BE, Frank LD. Healthy nutrition environments: concepts and measures. American Journal of Health Promotion 2005; 19(5): 330333.

5. Bezerra IN, Sichieri R. Características e gastos com alimentação fora do domicílio no Brasil. Rev Saude Publica 2010; 44(2):221-229.

6. Instituto Brasileiro de Geografia e Estatística (IBGE). Pesquisa de Orçamentos Familiares 2017-2018: primeiros resultados. Coordenação de Trabalho e Rendimentos. Rio de Janeiro: IBGE; 2019. 69p

7. Bleil SI. O Padrão Alimentar Ocidental: considerações sobre a mudança de hábitos no Brasil. Rev Cadernos de Debate 1998; 6(1):1-25.

8. Brasil. Ministério da Saúde. Secretaria de Atenção à Saúde. Guia alimentar para $a$ população brasileira. Brasília; 2014

9. Lima, B. T. A. N., Borges, A. F. Alimentação fora do lar: fatores que influenciam consumidores a optarem por um restaurante fast-food. Cad Prof Mark UNIMEP I. 2020; 8(1):1-18.

10. Martins APB, Levy RB, Claro RM, Moubarac JC, Monteiro CA. Participação crescente de produtos ultraprocessados na dieta brasileira (1987-2009). Rev Saude Publica 2013; 47(4):656-665. 
11. Zong G, Eisenberg DM, Hu FB, Sun Q. Consumption of meals prepared at home and risk of type 2 diabetes: an analysis of two prospective cohort studies. PLOS Medicine 2016; 13(7):e1002052.

12. Lytle LA. Measuring the Food Environment: State of the Science. Am J Prev Med 2009; 36(4):S134-S144.

13. Alves HJ, Boog MCF. Comportamento alimentar em moradia estudantil: um espaço para promoção da saúde. Rev Saude Publica 2007; 41(2):197-204.

14. Instituto Brasileiro de Geografia e Estatística (IBGE). Cidades 2020. Disponível em: https://cidades .ibge.gov.br/brasil/mg/juiz-de fora/ panorama. Acessado em: 27 mar. 2020.

15. Stamm C, Staduto JAR, Lima JF, Wadi YM. A população urbana e a difusão das cidades de porte médio no Brasil. Interações (Campo Grande) 2013; 14(2):251-265. <http://www.interacoes.ucdb.br/article/view/210>

16. Universidade Federal de Juiz de Fora (UFJF). Acesso a informação [sítio virtual] 2020 Disponível em: http://www.ufjf.br/ufjf/acesso-a-informacao/alunos/. Acessado em: 27 mar. 2020.

17. Leite FHM, Oliveira MAD, Cremm EDC, Abreu DSCD, Maron LR, Martins PA. Availability of processed foods in the perimeter of public schools in urban areas. J Pediatr (Rio J) 2012; 88(4):328-334.

18. Willett W, Skerrett PJ, Giovannucci EL, Callahan M. Eat, drink, and be healthy: the Harvard Medical School Guide to Healthy Eating. New York: Simon \& Schuster Source; 2001.

19. Monteiro CA, Cannon G, Levy RB, Moubarac J-C, Jaime P, Martins AP, Canella D, Louzada ML, Parra D. NOVA. A estrela brilha. Classificação dos alimentos. Saúde Pública. World Nutrition 2016; 7(1-3):28-40.

20. Cohen JA. Power primer. Psychological Bulletin 1992; 112(1):155-159.

21. Morland K, Wing S, Roux AD, Poole C. Neighborhood characteristics associated with the location of food stores and food service places. American Journal of Preventive Medicine 2002; 22(1): 23-29.

22. Walker RE, Keane CR, Burke JG. Disparities and access to healthy food in the United States: A review of food deserts literature. Health \& Place 2010; 16(5): 876-884.

23. Machado PP, Claro RM, Canella DS, Sarti FM, Levy RB. Price and convenience: The influence of supermarkets on consumption of ultra-processed foods and beverages in Brazil. Appetite 2017; 116: 381-388.

24. Laska MN, Hearst MO, Lust K, Lytle LA, Story M. How we eat what we eat: identifying meal routines and practices most strongly associated with healthy and unhealthy dietary factors among young adults. Public Health Nutrition 2015; 18(12): 2135-2145.

25. Mackay S, Vandevijvere S, Xie P, Lee A, Swinburn B. Paying for convenience: comparing the cost of takeaway meals with their healthier home-cooked counterparts in New Zealand. Public Health Nutrition 2017; 1-8.

26. Berti TL, Rocha TF, Curioni CC, Verly Junior E, Bezerra FF, Canella DS et al . Consumo alimentar segundo o grau de processamento e características 
sociodemográficas: Estudo Pró-Saúde. Rev Bras Epidemiol [Internet] 2019; 22: (e190046):1-14.

27. Pulz IS. Ambiente Alimentar do Campus Sede da Universidade Federal de Santa Catarina [dissertação]. Florianópolis: Programa de Pós-Graduação em Nutrição da Universidade Federal de Santa Catarina; 2014.

28. Popkin BM, Duffey K, Gordon-Larsen P. Environmental influences on food choice, physical activity and energy balance. Physiology \& Behavior 2005; 86(5):603-613.

29. Azevedo IM. Fatores relevantes do comportamento alimentar: uma pesquisa com estudantes universitários. Rev Soc Dev 2019; 8(5):e39855761.

30. Byrd-Bredbenner C, Johnson M, Quick VM, Walsh J, Greene GW, Hoerr S, Horacek TM. Sweet and salty. An assessment of the snacks and beverages sold in vending machines on US post-secondary institution campuses. Appetite 2012; 58(3):1143-1151.

31. Tomer JF. Stemming the tide of obesity: What needs to happen. J Socio Econ 2013; 42:88-98.

32. Nordin SM, Boyle M, Kemmer TM. Position of the Academy of Nutrition and Dietetics: Nutrition security in developing nations: Sustainable food, water, and health. J Acad Nutr Diet 2013; 113(4):581-595

Submissão: $27 / 03 / 2020$

Aprovação: 12/08/2020 